\title{
BRIGHT IDEAS PROBLEM-SOLVING SKILLS TRAINING FOR CAREGIVERS OF CHILDREN WITH SICKLE CELL DISEASE: A TWO-SITE PILOT FEASIBILITY TRIAL
}

\author{
Melissa Young ${ }^{1}$, Megan Voll ${ }^{2}$, Robert Noll ${ }^{3}$, Diane Fairclough ${ }^{4}$, and Catherine \\ Flanagan-Priore ${ }^{5}$ \\ ${ }^{1}$ Hospital for Sick Children \\ ${ }^{2}$ University of Pittsburgh \\ ${ }^{3}$ Children's Hospital of Pittsburgh \\ ${ }^{4}$ Colorado School of Public Health \\ ${ }^{5}$ John R Oishei Children's Hospital
}

June 10, 2020

\begin{abstract}
Bright IDEAS Problem-Solving Skills Training (BI) is an evidence-based behavioral intervention that has been utilized extensively with caregivers of children recently diagnosed with cancer. Considerable evidence has shown that BI is acceptable to caregivers and improvements in problem-solving skills mediate reduced symptoms of distress; and it is most effective with single, minority caregivers. A slightly modified version of BI was offered to caregivers of children with sickle cell disease (SCD) in a two-site pilot feasibility trial. BI was modified to reduce barriers to care, logistical challenges, and stigma associated with receiving behavioral health services. Our goal was to establish high rates of recruitment and retention amongst caregivers of children with SCD. Recruitment was acceptable $(94 \% ; \mathrm{N}=72)$ and retention reasonable $(48.6 \%)$ across both sites with 35 caregivers successfully completing the BI program. Results showed that caregivers of children with SCD, who successfully completed the BI program reported, significant improvements in problem-solving skills immediately and three months post-intervention completion. Interestingly, initial levels of distress were low with few caregivers reporting clinically significant levels of distress; distress remained low over time. Findings are discussed in the context of psychosocial screening and assumptions regarding caregivers of children with SCD.
\end{abstract}

\section{INTRODUCTION}

African American and minority caregivers continue to have an overrepresentation of poverty in comparison to other demographic groups living in the United States (US) ${ }^{1,2}$. Sickle cell disease (SCD) is a genetic hematological disorder that disproportionately impacts African American individuals in the US ${ }^{1,3}$. According to the Centers for Disease Control and Prevention ${ }^{4}$, SCD affects approximately 100,000 Americans; 1 of every 365 African American newborn children have the disease, as well as 1 of every 16,300 Hispanic-American children within the US. Complications of SCD can result in chronic and/or life-threatening sequalae due to vaso-occlusive crises, bacterial infections, cerebral infarctions, and chronic anemia ${ }^{5,6,7,8,9}$.

Considering the chronicity, medical complexity, and potential severity of symptoms in children with SCD, it is feasible that caring for a child with SCD may have adverse psychosocial effects on caregivers, the child with SCD, and the broader family system ${ }^{10}$. Notably, some studies report caregivers of children with SCD 
experience significant levels of distress ${ }^{11,12,13,14}$ whereas other studies report levels of distress comparable to other minority caregivers who live in the same neighborhoods but do not have a child with a chronic illness ${ }^{15,16}$.

Barakat and colleagues examined the relationships between parental problem-solving abilities, disease-related complications in the child with SCD (from the medical record), and the child's self-reported health-related quality of life (HRQOL) ${ }^{17}$. Findings suggested that parental problem-solving skills moderated the relationship between disease-related complications and the psychosocial HRQOL reported by the child with SCD.

With consideration of the findings from Barakat ${ }^{17}$ and the known challenges associated with raising a child with SCD, we sought to deliver an evidence-based intervention, Bright IDEAS Problem-Solving Skills Training (BI) to caregivers of these children. BI was originally designed to assist caregivers (i.e., primarily mothers) of children recently diagnosed with cancer. Further, BI was developed to improve problem-solving skills of caregivers with the expectation that improvements in problem-solving skills would lead to (mediate) reductions in distress ${ }^{18,19,20}$. We endeavored to deliver BI to caregivers of children with SCD, with the same focus of improving problem-solving skills, anticipating that improved coping skills would lead to less distress. To our knowledge, this was the first attempt to adapt and utilize an evidence-based intervention, designed to improve problem-solving skills and alleviate distress, with caregivers of children with SCD.

The purpose of this two-site pilot feasibility study was to modify the delivery of BI to reduce barriers to care and stigma surrounding behavioral healthcare for this population of caregivers. We appreciated that recruitment and retention of minority caregivers in clinical research has historically and continues to be a challenge $\mathrm{e}^{2,21,22}$. We anticipated that our delivery modifications could improve rates of recruitment and retention. We also expected that caregivers of children with SCD who received BI would report improved problem-solving skills and lower levels of distress immediately following completion of BI and three months post-completion of BI compared to baseline.

\section{Methods}

\section{Participants}

Caregivers of children with SCD were recruited from two comprehensive pediatric SCD centers (Buffalo and Pittsburgh). Eligibility criteria were: (1) primary caregiver of a child, from birth through 10 years of age, diagnosed with any type of SCD; (2) English-speaking; and (3) residing within 30 miles of the respective study site.

\section{Procedures}

Medical care teams were informed about BI and previous research demonstrating success (acceptability and effectiveness) amongst caregivers of pediatric oncology patients. Medical providers then approached eligible caregivers during the child's routine outpatient hematology appointments and/or inpatient admissions, to obtain caregivers permission to be contacted regarding their interest in this project (Figure 1). After obtaining consent, caregivers were asked to complete baseline assessments (T1) and a time was scheduled to start BI sessions.

\section{B right IDEAS Problem-Solving Skills Training}

$\mathrm{BI}$ is an evidence-based manualized intervention, based foundationally on the tenets of Problem-Solving Therapy $^{23}$. In previously published work, the intervention was titled Problem-Solving Skills Training ${ }^{18,19}$ (PSST); however, during an ongoing dissemination science grant, professionals trained to deliver the intervention, as well as patient advocates, suggested that relabeling the intervention (i.e., Bright IDEAS rather than PSST) would make it more acceptable to families. In addition, professionals and advocates felt the Bright IDEAS label was a more accurate depiction of the goals of the intervention. 
Bright signifies the concept of optimism (i.e., positive problem orientation - You can do it ), which is essential to successful problem-solving ${ }^{24}$. 'IDEAS' is a mnemonic for each of the BI problem-solving steps with each letter signifying a step in the problem-solving process: I (Identify the problem), D (Determine all possible options), E (Evaluate your options - pros and cons of each option), A (Act - create an action plan based on $\mathrm{D}$ and $\mathrm{E}$ ), and S (See if it worked - if the plan does not work go back to steps D and E respectively).

During BI sessions, participants are taught to utilize a five-step approach to problem-solving ${ }^{18,19,20}$ (Figure 2 ). Over the past 25 years of developing and assessing the effectiveness of BI, a comprehensive instructor's manual (Supplement 1: Instructor's Manual) was developed to serve as a guide for therapists when delivering the intervention to caregivers. It was not changed for this research. For this study, we modified the Cancer Parent Manual previously developed for caregivers of children with cancer, to include relevant information for caregivers of children with SCD (Supplement 2: SCD Parent Manual). We also used BI worksheets (Supplement 3: Worksheets) during sessions, as these materials contain attractive graphics and simplify the problem-solving process for caregivers. The worksheets were not changed for this research.

BI was presented to caregivers as a systematic approach that could be used to overcome any life challenge, including those commonly faced when caring for a child diagnosed with SCD. Following our template for providing BI to caregivers of children with cancer, BI was delivered over the course of 6-8 individual sessions, with each session following a structured format, and lasting approximately 30-60 minutes. During the first session therapists focused on: establishing rapport; learning about the family and the child's medical history; and explaining BI. Caregivers were also provided a copy of the SCD Parent Manual and Worksheets. During subsequent sessions (i.e., sessions 2-8) therapists focused on: reviewing the BI program and worksheets; assisting caregivers with identifying challenges; systematically guiding caregivers through the problem-solving steps using the 'IDEAS' mnemonic; and during the final session the principles of the program were reviewed and strategies for relapse prevention were discussed.

Although the content of BI was identical to previous efforts with PSST, in the current study, delivery times and locations were markedly more flexible and determined based on the caregiver's preference and needs to reduce the impact of logistical barriers (e.g., lack of transportation, reliable childcare, etc.) on participation. In prior BI studies within pediatric oncology, sessions were primarily conducted during the child's hospitalizations or clinic visits. In the present study, our flexible community-based approach was modeled after BI work with caregivers of children recently diagnosed with autism ${ }^{25}$. BI sessions were completed in a multitude of settings including home visits, meeting at the caregiver's work setting during lunch, Skype, and so on. Not only could caregivers choose the location of each session, but they could also select any time that worked for them, which included evening and weekend sessions. Our ultimate goal was to make BI as accessible as possible. Participants were also encouraged to use the BI Worksheets between sessions (i.e., homework), but this was not required.

\section{Measures}

Measures were administered at three time points: (a) at baseline prior to receiving BI (T1); (b) immediately following completion of 6-8 BI sessions (T2); and three months following completion of BI sessions (T3). Demographic information was only obtained at T1. Of note, attempts were made via phone and/or email to obtain T2 and T3 data from caregivers who did not complete the BI program.

\section{Demographics}

The demographic questionnaire included basic information about the caregiver, the child with SCD, and the family system. Questions focused on caregiver education, occupation, marital status, etc. Caregiver socioeconomic status was determined using occupation and education ${ }^{28,29}$.

\section{Social Problem-Solving Inventory-Revised (SPSI-R)}

The SPSI-R is a 52 -item self-report measure designed to assess an individual's ability to solve problems ${ }^{30}$. SPSI-R statements are endorsed by respondents on a 5-point likert scale ranging from 1 (i.e., not true at all) 
to 5 (i.e., extremely true). The SPSI-R assesses positive problem orientation (PPO) and rational problem solving (RPS), which are considered constructive/adaptive problem-solving dimensions. Negative problem orientation (NPO), impulsivity/carelessness style (ICS), and avoidance style (AS) are also measured and categorized as dysfunctional problem-solving dimensions. A total score (0-20) is calculated using the five subscores with consideration of whether the sub-scores are constructive/adaptive or dysfunctional dimensions. Higher total scores indicate that the individuals utilize constructive problem-solving orientation and rational problem-solving styles more frequently. The SPSI-R was used as a primary measure of intervention efficacy. In previous research, the internal consistency of the SPSI-R was found to be adequate when used with parents of children with SCD (Cronbach's $\alpha=0.86$ ). ${ }^{17}$ In the current study, the Cronbach's $a$ for the SPSI-R total score was .87. Cronbach's $a$ for the SPSI-R subscales were as follows; PPO .80, NPO .87, RPS .95, ICS .79, and AS .78.

\section{Patient Health Questionnaire-9 (PHQ-9)}

The PHQ-9 is a 9-item self-report measure of depressive symptoms ${ }^{31}$. Each of the 9 items are rated on a 4-point likert scale ranging from 0 (i.e., not at all) to 3 (i.e., nearly every day) with respondents required to rate the frequency of the depressive symptoms experienced within the last two weeks. Total scores range from 0 (no symptoms of depression) to 27 (i.e., severe depression). A score of 15 and above is suggestive of clinical depression. The PHQ-9 was used as a secondary measure of intervention efficacy. When used with an older African American population the PHQ-9 Cronbach's $\alpha$ was found to be $0.75^{32}$. In the current study, the PHQ-9 Cronbach's $a$ was .86.

\section{Profile of Mood States (POMS)}

The POMS is a 65-item self-report measure consisting of seven self-report rating scales about feelings experienced over the previous week ${ }^{33,34}$. In the current study, we used a 15-item short-form of the POMS, as in prior BI studies ${ }^{35}$. The POMS items were rated from 0 (not at all) to 4 (extremely). The POMS produces seven subscales: (1) tension-anxiety, (2) depression-dejection, (3) anger-hostility, (4) fatigue-inertia, (5) confusion-bewilderment, (6) vigor-activity, and (7) friendliness. The subscale scores were combined to create a total mood disturbance (TMD) score. The POMS was used as a secondary measure of intervention efficacy. In the current study, the POMS TMD Cronbach's $a$ in the current sample was .85 .

\section{Impact of Events Scale-Revised (IES-R)}

The IES-R is a 22-item self-report questionnaire that includes three subscales (i.e., hyperarousal, intrusion, and avoidance) that are associated with post-traumatic stress disorder (PTSD) ${ }^{36}$. Items are rated on a 4-point scale, based on frequency of occurrence, ranging from "not at all" to "often." Higher scores indicate more symptoms of post-traumatic stress disorder. The IES-R was used as a secondary measure of intervention efficacy. The IES-R IES-R test-retest reliability estimates obtained from African American breast cancer survivors ranged from 0.89 to 0.94 over a 6 -month study period ${ }^{37}$. In the current study, the Cronbach's $a$ for this sample was .93.

\section{Data Analysis}

Descriptive statistics are provided for the demographic data. We anticipated that caregivers would report higher levels of problem-solving skills and lower levels of distress at T2 and T3 compared to T1. To analyze the changes in problem-solving skills and distress over time, the SPSI-R, PHQ-9, POMS, and IES-R raw scores were analyzed using Maximum Likelihood Estimation for unbalanced (i.e., incomplete) repeated measures. As in prior BI studies ${ }^{18,19,20}$, this approach was chosen to avoid making the assumption that data are missing-completely-at-random, which occurs with case deletion when using traditional multivariate analysis of variance. Thus, all caregivers, regardless of whether they completed BI, were included in the "intent-totreat" analyses. In addition, because we had clear directional expectations, one-tailed t-tests are reported. All data analyses were completed using IBM SPSS Statistics Premium Grad Pack 25 for Mac.

\section{Results}




\section{Demographics}

The majority of the caregivers were single (48.3\%), female (67\%), African American (83.3\%), and reported to be working in 'blue collar' occupations (Table 1). The average SES score (Entwisle \& Astone, 1994) for the caregivers $(\mathrm{N}=60)$ was 29.7 (e.g., hairdressers, health aides, etc.) with a range from 15.0 (i.e., unemployed) to 73.2 (i.e., registered nurse). The average SES score for the caregivers' spouses $(\mathrm{N}=38)$ was 35.98 with a range from 15.0 (i.e., unemployed) to 76.31 (i.e., computer programmers).

\section{Recruitment and Retention}

We were given permission by the medical teams to approach 77 caregivers of children with SCD and 72 (94\%) consented to participate (Figure 1). After consenting 72 caregivers, 60 completed T1 assessments (83\%). Of the 72 caregivers who consented, 26 (36\%) completed 0 sessions; 16 participants (22\%) completed 1-5 sessions; and 35 participants $(48.6 \%)$ completed $6+$ sessions. T2 data were provided by 39 participants $(54.2 \%)$ and 41 participants completed T3 (56.9\%). Of note, there were no statistically significant differences in recruitment and retention between the two study sites.

\section{Problem-Solving Skills}

SPSI-R scores consistently improved over time (Table 2). Data show that standard scores on the SPSI-R and PPO, NPO, RPS, ICS, and AS subscales were all within one standard deviation $(\mathrm{SD}=15)$ of the mean $(\mathrm{M}=100)$ based on normative data (Table 2$)$ at all assessment points ${ }^{30}$.

\section{Distress}

In clinical settings, t-scores greater than 70 on the POMS TMD are considered very elevated, t-scores between 60 and 69 are elevated, t-scores between 40 and 59 are average, t-score between 30 and 39 are low, and t-scores below 30 are very low $^{33}$. The POMS TMD t-scores show that caregivers of children with SCD reported non-clinical levels of mood disturbance at $\mathrm{T} 1, \mathrm{~T} 2$, and $\mathrm{T} 3$, as all scores were within the average range (Table 3$)^{33}$.

The PHQ-9 data suggests that caregivers of children with SCD in this sample reported few symptoms of depression at T1, T2, and T3 (Table 4). Levels of depression among 9 (15\%) caregivers were within the mild depression range (scores $10-14) ; 4(6.7 \%)$ caregivers were within the moderate depression range (scores 17 $-19)^{38}$; none reported severe depression (score 20+).

On average, IES-R scores were within the non-clinical range at T1, T2, and T3 (Table 4$)^{36}$. In clinical settings, a score of 24 or higher on the IES-R is of clinical concern and warrants further assessment ${ }^{36}$.

\section{Effectiveness of Intervention for Caregivers who Completed 6+ Sessions}

To further explore our data, we also examined the effectiveness of the BI intervention for the 35 caregivers who successfully completed $6+$ sessions. For this group of 35 caregivers, SPSI-R scores improved consistently over time. Scores on the POMS TMD, PHQ-9, and IES-R were low initially, and they remained low at all three time points.

\section{DISCUSSION}

The purpose of the present study was to modify the delivery of BI to caregivers of children with SCD in ways that would increase accessibility and reduce stigma associated with receiving behavioral health services. We anticipated that delivery modifications could improve rates of recruitment and retention from previous attempts to recruit caregivers of children with SCD for participation in psychosocial research. With recruitment and delivery modifications (described above), 94\% of caregivers of children with SCD consented 
to participate and $48.6 \%$ completed $6+$ sessions of face-to-face BI. These rates of recruitment and retention were higher than those reported in other studies of children with SCD suggesting that our strategies to reduce barriers to participation were moderately successful for this group of caregivers ${ }^{17}$.

We anticipated that caregivers of children with SCD who successfully completed the BI program (6+ sessions), would report improvements in problem-solving skills and lower levels of distress between T1 and T2 and also between $\mathrm{T} 1$ and $\mathrm{T} 3$. Based on our clinical experience and the behavioral health literature regarding families of children with SCD (i.e., minority, single parents, low SES), we initially believed these caregivers would report below average levels of problem-solving skills and considerable levels of distress at T1 due to the complexity of caring for a child with SCD and challenges associated with being a minority parent. Our data showed that caregivers of children with SCD reported average levels of problem-solving skills and non-clinical levels of distress at T1, T2, and T3.

While the majority of our sample of caregivers were low SES, single, minority parents who were caring for a child with a severe, chronic illness, our data suggested that these caregivers of children with SCD experience characteristics of psychological hardiness that we did not anticipate. We suspect our belief that caregivers of children with SCD would report below average levels of problem-solving skills and higher levels of distress, in comparison to other populations of caregivers, may have been attributable to existing research demonstrating significant levels of parenting stress and other psychosocial challenges, as well as our own intrapersonal professional biases, as we did not initially consider the possibility that caregivers of children with SCD may have solid problem-solving skills and little evidence of distress at baseline ${ }^{12,16,17,39}$. Thus, T1 data from the present study suggest that as a group, caregivers of children with SCD who participated in this study have broadly average problem-solving skills and they are experiencing non-clinical levels of distress.

Future research with caregivers of children with SCD might focus on families who score within the targeted or clinical level for psychosocial risk, which could be accomplished through comprehensive screening efforts utilizing the Psychosocial Assessment Tool $(\mathrm{PAT})^{40}$. Prior investigations of the PAT with families of children with SCD, demonstrated increased risk for parental stress when the child with SCD did not have government health benefits, lower caregiver education, more children residing within the home, and greater financial difficulties $^{40}$. Thus, identifying and targeting caregivers for participation in BI, who report higher levels of overall psychosocial risk on the PAT, may produce more robust results with regards to the effectiveness of the intervention with this population of caregivers.

Although we demonstrated successful recruitment of caregivers of children with SCD, levels of retention in this study were modest. In light of the rapidly evolving use of telehealth and iPhone/Android application-based psychosocial intervention programs, researchers may consider utilizing electronic mediums for disseminating BI-content. However, recent work comparing face-to-face BI to BI provided entirely via a website showed that face-to-face delivery was more effective ${ }^{41}$. With consideration of these findings ${ }^{41}$, future researchers may consider using the website-BI program as an adjunct to face-to-face sessions in order to further improve accessibility of the BI intervention for caregivers.

Our study had several limitations. First, our sample size of caregivers of children with SCD was small and underpowered to detect small or medium differences. Second, the study design did not include a control group of caregivers of children with SCD who did not receive BI. Third, although we recruited caregivers from two sites, it is possible that our sample of caregivers is not representative of the larger population of caregivers of children with SCD. Thus, future studies designed to implement a psychosocial intervention with caregivers of children with SCD, may benefit from collaborating with multiple sites (i.e., $3+$ ), similar to prior large-scale randomized controlled trials of BI in pediatric oncology, to increase the sample size and thus statistical power ${ }^{18,19,20}$. We also strongly recommend screening caregivers, who report low levels of problem-solving skills and/or high levels of distress, prior to delivery to ascertain those in highest need.

In summary, data obtained from the present study provides limited evidence to support further efforts in designing and conducting a randomized control trial (RCT) of $\mathrm{BI}$ for this population of caregivers. However, 
focusing future efforts on identifying caregivers with the highest risk (i.e., low levels of problem-solving skills and high levels of distress) based on screening and subsequently delivering BI may be of clinical benefit.

Conflict of Interest Statement

Melissa A. Young, PsyD: No conflicts of interest to declare.

Megan Voll, LPC: No conflicts of interest to declare.

Robert B. Noll, PhD: No conflicts of interest to declare.

Diane L. Fairclough DrPH: No conflicts of interest to declare.

Cate Flanagan-Priore, PhD: No conflicts of interest to declare.

This work was supported by a grant to RBN from the National Center for Advanced Translational Sciences Grant Number UL1 TR001857-02.

References

1. Edwards CL, Scales MT, Loughlin C, Bennett GG, Harris-Peterson S, DeCastro LM, Whitworth E, Abrams M, Feliu M, Johnson S, Wood M, Harrison O, Killough A. A brief review of the pathophysiology, associated pain, and psychosocial issues in sickle cell disease. Int J Behav Med 2005; 12: 171-179.

2. Hankins J, Wang W. The painful face of poverty. Pediatr Blood Cancer 2009; 52:157-158.

3. Ware RE, De Montalembert M, Tshilolo L, Abboud MR. Sickle cell disease. Lancet 2017; 390: 311-323.

4. Centers for Disease Control and Prevention Website. https://www.cdc.gov/ncbddd/sicklecell/data.html Accessed August 9, 2017.

5. Chaturvedi S, Debaun MR. Evolution of sickle cell disease from a life-threatening disease of children to a chronic disease of adults: The last 40 years. Am J Hematol 2015; 91: 5-14.

6. Esezobor CI, Akintan P, Nwaogazie U, Akinwunmi E, Temiye E, Akinsulie A, Gbadegesin R. Enuresis in children and adolescents with sickle cell anaemia if more frequent and substantially different from the general population. PLoS One 2018; 8: 1-12.

7. Iampietro M, Giovannetti T, Tarazi R. Hypoxia and inflammation in children with sickle cell disease: Implications for hippocampal functioning and episodic memory. Neuropsychol Rev 2014; 24: 252-265.

8. Lawrence C, Webb J. Sickle cell disease and stroke: Diagnosis and management. Curr Neurol Neurosci Rep 2016; 16: 1-10.

9. Platt OS, Rosse WF, Milner PF, Castro O, Steinberg MH, Mortality in sickle cell disease. Life expectancy and risk factors for early death. N Engl J Med 1994; 330: 1639-1644.

10. Amid A, Odame I. Improving outcomes in children with sickle cell disease: Treatment considerations and strategies. Paediatr Drugs 2014; 16: 255-266.

11. Barakat LP, Patterson CA, Tarazi RA, Ely E. Disease-related parenting stress in two sickle cell disease caregiver samples: Preschool and adolescent. Fam Syst Health 2007; 25: 147-161.

12. Hildenbrand AK, Barakat LP, Alderfer MA, Marsac ML. Coping and coping assistance among children with sickle cell disease and their parents. J Pediatr Hem Onc 2015; 37: 25-34.

13. Hofmann M, Montalembert M, Beauquier-Maccotta B, Villartay P, Golse B. Posttraumatic stress disorder in children affected by sickle-cell disease and their parents. Am J Hematol 2007; 82: 171-172.

14. Northington L. Chronic sorrow in caregivers of school age children with sickle cell disease: A grounded theory approach. Issues Compr Pediatr Nurs 2000; 23: 141-154.

15. Noll R, Swiecki E, Garstein M, Vannatta K, Kalinyak KA, Davies W, Bukowski W. Parental distress, family conflict, and role of social support for caregivers with or without a child with sickle cell disease. Fam Syst Med 1994; 12: 281-294.

16. Thompson RJ, Gil KM, Gustafson KE, George LK, Keith BR, Spock A, Kinney TR. Stability and change in the psychological adjustment of mothers of children and adolescents with cystic fibrosis and sickle cell disease. J Pediatr Psychol 1994; 19: 171-188.

17. Barakat LP, Daniel LC, Smith K, Robinson MR, Patterson CA. Parental problem-solving abilities and the association of sickle cell disease complications with health-related quality of life for school-age 
children. J Clin Psychol Med Settings 2014; 21: 56-65.

18. Sahler OJZ, Dolgin MJ, Phipps S, Fairclough DL, Askins MA, Katz ER, Noll RB, Butler RW. Specificity of problem-solving skills training in mothers of children newly diagnosed with cancer: Results of a multisite randomized clinical trial. J Clin Oncol 2013; 31: 1329-1335.

19. Sahler OJZ, Fairclough DL, Phipps S, Mulhern RK, Dolgin MJ, Noll RB, Katz ER, Varni JW, Copeland DR, Butler RW. Using problem solving skills training to reduce negative affectivity in mothers of children with newly diagnosed cancer: Report of a multisite randomized trial. J Clin Coun Psychol 2005; 73: 272-283.

20. Sahler OJZ, Varni JW, Fairclough DL, Butler RW, Noll RB, Dolgin MJ, Phipps D, Copeland DR, Katz ER, Mulhern RK. Problem-solving skills training for mothers of children with newly diagnosed cancer: A randomized trial. J Dev Behav Pediatr 2002; 23: 77-86.

21. Gamble VN. Under the shadow of Tuskegee: African Americans and healthcare. Am J Hematol 1977; 87: 1773-1778.

22. Stevens EM, Patterson CA, Li YB, Smith-Whitley K, Barakat LM. Mistrust of pediatric sickle cell disease clinical trials research. Am J Prev Med 2016; 51: S78-S86.

23. Nezu AM. Efficacy of a social problem-solving therapy approach for unipolar depression. J Clin Coun Psychol 1986; 54: 196-202.

24. Nezu AM, Nezu CM, D'Zurilla TJ. Problem-solving therapy: A treatment manual. New York, NY: Springer Publishing Company, LLC: 2013.

25. Nguyen CT, Fairclough DL, Noll RB. Problem-solving skills training for mothers of children recently diagnosed with autism spectrum disorder: A pilot feasibility study. Autism 2016; 20: 55-64.

26. Kelly AD, Egan AM, Reiter-Purtill J, Gerhardt CA, Vannatta K, Noll RB. A controlled longitudinal study of internalizing symptoms in older adolescents with sickle cell disease. Pediatr Blood Cancer 2015; 62: 637-642.

27. Noll RB, Kiska R, Reiter-Purtill J, Gerhardt CA, Vannatta K. A controlled longitudinal study of the social functioning of adolescents with sickle cell disease. Pediatrics 2010; 125: e1453-e1459.

28. Entwisle DR, Astone NM. Some practical guidelines for measuring youth's race/ethnicity and socioeconomic status. Child Dev 1994; 65: 1521-1540.

29. Shavers VL. Measurement of socioeconomic status in health disparities research. J Natl Med Assoc 2007; 99: 1013-1023.

30. D’Zurilla TJ, Nezu AM, Maydeu-Olivares A. Social problem-solving inventory-Revised. North Tonawanda, NY: MHS; 2002.

31. Bian C, Li C, Duan Q, Wu H. Reliability and validity of patient health questionnaire: Depressive syndrome module for outpatients. Sci Res Ess 2011; 6: 278-282.

32. Gitlin LN, Chernett NL, Dennis MP, Hauck WW. Identification of and beliefs about depressive symptoms and preferred approaches among community-living older African Americans. Am J Geriatr Psychiatry 2012; 20: 973-984.

33. Heuchert JP, McNair DM. Profile of mood states - Second edition. Canada, Multi-Health Systems; 2012 .

34. McNair DM, Lorr M, Doppleman LF. Manual for the profile of mood states. San Diego, CA: Educational and Industrial Testing Services; 1971.

35. Steele RG, Long A, Reddy KA, Luhr M, Phipps S. Change in maternal distress and child-rearing strategies across treatment for childhood cancer. J Pediatr Psychol 2002; 28: 447-462.

36. Weiss DS. The impact of event scale - revised. Assessing Psychological Trauma and PTSD: A Practitioner's Handbook $-2^{\text {nd }}$ Edition. New York, NY: Guilford Press; 2007.

37. Von DM, Russell KM, Carpenter J, Monahan PO, Zhao Q, Tallman E, Ziner KW, Storniolo AM, Miller KD, Giesler RB, Haase J, Otte J, Champion VL. Health-related quality of life of African American breast cancer survivors compared to healthy African American women. Cancer Nurs 2012; 35: 337-346.

38. Kroenke K, Spitzer R, Williams W. The patient health questionnaire PHQ-9: Validity of a brief depression severity measure. J Gen Int Med 2001; 16: 2-3.

39. Thompson R, Gil K, Burbach D, Keith, B, Kinney T. Psychological adjustment of mothers of children 
and adolescents with sickle cell disease: The role of stress, coping methods, and family functioning. J Pediatr Psychol 1993; 18: 549-559.

40. Karlson CW, Leist-Haynes S, Smith M, Faith MA, Elkin TD, Megason G. Examination of risk and resiliency in a pediatric sickle cell disease population using the psychosocial assessment tool 2.0. J Pediatr Psychol 2012; 37: 1031-1040.

41. Phipps S, Fairclough DL, Noll RB, Devine KA, Dolgin MJ, Schepers SA, Askins MA, Schneider N, Ingman K, Voll M, Katz ER, McLaughlin J, Sahler OJZ. In-person vs. web-based administration of a problem-solving skills intervention for parents of children with cancer: Report of a randomized non-inferiority trial. E Clinical Medicine, Published by THE LANCET, in press.

Legends

TABLE 1 Demographic Characteristics of Participants $(n=60)$ and their Spouses $(n=38)$

TABLE 2 Comparison of Problem-Solving Skills Standard Score Changes at Baseline (T1) to T2 and T1 to $\mathrm{T} 3$

TABLE 3 Comparison of Mood Disturbance T-score Changes at Baseline (T1) to T2 and T1 to T3

TABLE 4 Comparison of Depression and Post-Traumatic Stress Symptoms Mean and Standard Deviation Changes at Baseline (T1) to T2 and T1 to T3

Figure 1 Consort Diagram

Figure 2 Bright IDEAS Model

\section{Hosted file}

Table 1_Demographics.docx available at https://authorea.com/users/331781/articles/458338bright-ideas-problem-solving-skills-training-for-caregivers-of-children-with-sicklecell-disease-a-two-site-pilot-feasibility-trial

\section{Hosted file}

Table 2_SPSIR.docx available at https://authorea.com/users/331781/articles/458338-brightideas-problem-solving-skills-training-for-caregivers-of-children-with-sickle-celldisease-a-two-site-pilot-feasibility-trial

\section{Hosted file}

Table 3_POMS.docx available at https://authorea.com/users/331781/articles/458338-brightideas-problem-solving-skills-training-for-caregivers-of-children-with-sickle-celldisease-a-two-site-pilot-feasibility-trial

\section{Hosted file}

Table 4_PHQ9_IESR.docx available at https://authorea.com/users/331781/articles/458338bright-ideas-problem-solving-skills-training-for-caregivers-of-children-with-sicklecell-disease-a-two-site-pilot-feasibility-trial 


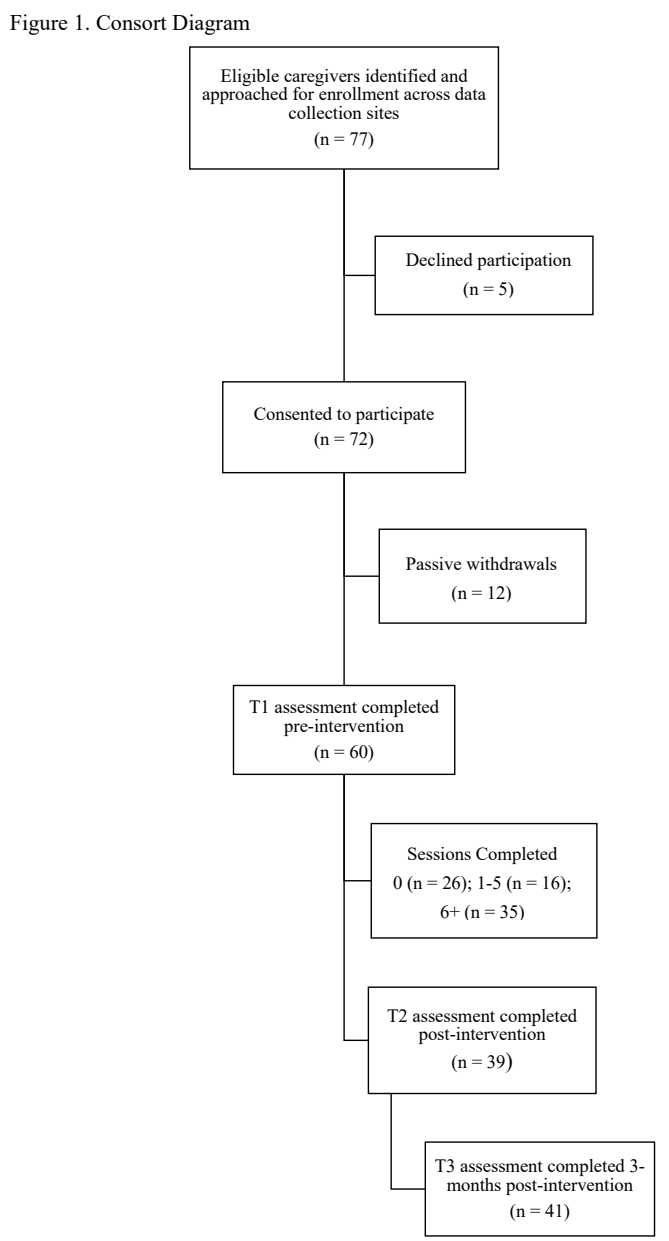

\section{Hosted file}

Figure 2_Bright IDEAS Model.doc available at https://authorea.com/users/331781/articles/ 458338-bright-ideas-problem-solving-skills-training-for-caregivers-of-children-withsickle-cell-disease-a-two-site-pilot-feasibility-trial 